\title{
糖基含磷配体在不对称氢化反应中的应用
}

\author{
崔朋雷* 刘卉敏果秀敏 张冬暖 王彦恩 王 春 \\ (河北农业大学理学院 保定 071001)
}

\begin{abstract}
摘要 不对称氢化反应在实际工业生产中有非常重要的应用. 糖类是广泛存在于自然界的手性天然产物, 手性糖类化 合物的合成与应用研究日益受到人们的关注，以糖类衍生物作为手性配体用于不对称反应已成为有机化学中非常活跃 的研究领域. 综述了糖基含磷配体用于不对称氢化反应的最新研究进展.
\end{abstract}

关键词 不对称氢化反应; 糖类; 手性配体

\section{Application of Sugar-Based Phosphorus Ligands in Asymmetric Hydrogenation}

\author{
Cui, Penglei* Liu, Huimin $\quad$ Guo, Xiumin $\quad$ Zhang, Dongnuan \\ Wang, Yanen Wang, Chun \\ (College of Science, Agricultural University of Hebei, Baoding 071001)
}

\begin{abstract}
There is a very important application for the asymmetric hydrogenation in the actual industrial production. Sugar is chiral natural product which widely exists in the nature. More and more attention was paid to the research of the chiral sugar's synthesis and application. Sugar derivatives, as chiral ligands, were widely used in asymmetric reactions and the relative research became a very active area in organic chemistry. The recent progress of sugar-based phosphorus-containing ligands that used in asymmetric hydrogenation is reviewed.

Keywords asymmetric hydrogenation; sugar; chiral ligand
\end{abstract}

不对称氢化反应在实际工业生产中有非常重要的 应用, 如在工业化合成左旋多巴 (又名左多巴, 为抗震 颤麻痹药)及手性农药异丙甲草胺的过程中都会用到不 对称催化氢化过程. 目前, 不对称催化氢化反应已成为 研究最为深入的不对称反应之一. 在不对称催化反应中 寻找新型更加有效的手性配体一直是人们研究的热点 问题. 糖类是广泛存在于自然界的手性天然产物, 近年 来糖类衍生物作为不对称合成的手性配体的应用日益 广泛. 糖类由于自身具有多官能团和多手性中心的结构 特点, 所以具有潜在的诱导手性的能力, 糖类衍生物作 为手性配体已经成功用在一些不对称合成中并且表现 出了良好的对映选择性 ${ }^{[14]}$. 糖基配体中往往以 $\mathrm{P}, \mathrm{N}$ 等 给电子原子作为配位原子与过渡金属形成络合物用于 不对称催化反应中具有良好的催化作用. 本文综述了糖 基含磷配体用于不对称氢化反应的最新研究进展.

\section{1 叔膦糖基配体}

在 20 世纪 70,80年代,一些含磷的糖基配体被设计 合成出来 ${ }^{[5 \sim 18]}$, 但是只有配体 $\mathbf{1}^{[7]}$ 及配体 $\mathbf{2}^{[16]}$ (Scheme 1) 在不对称氢化反应中具有较高的对映选择性.
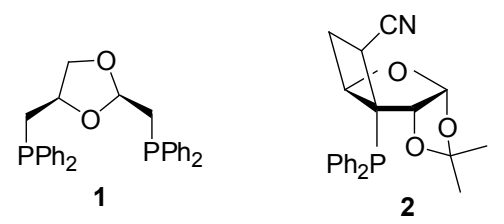

Scheme 1

后来人们又合成了一系列含有呋喃糖苷骨架的二 膦配体 3 5 (Scheme 2), 这些配体被用于 $\mathrm{Rh}$ 催化的 $\alpha, \beta$-不饱和羧酸的不对称氢化反应, 结果发现产物的 $e e$ 值最高可达 $98 \%{ }^{[19,20]}$. 实验结果也发现在配体 3 结构中 C-5 上引入甲基可以使其活性显著增加, 并且配体中 C-5 的构型会对反应产物的对映选择性产生强烈的影响

*E-mail: 936369081@qq.com

Received September 14, 2012; revised November 13, 2012; published online November 20, 2012. 
(配体 4 中 C-5 为 $R$ 构型, 而配体 5 中 C-5 为 $S$ 构型, 配 体 4 在反应中表现出更高的对映选择性). Imamoto 等 ${ }^{[21]}$ 对于含磷配体与 $\mathrm{Rh}$ 催化的不对称氢化反应的机理进行 了探讨(Scheme 3).
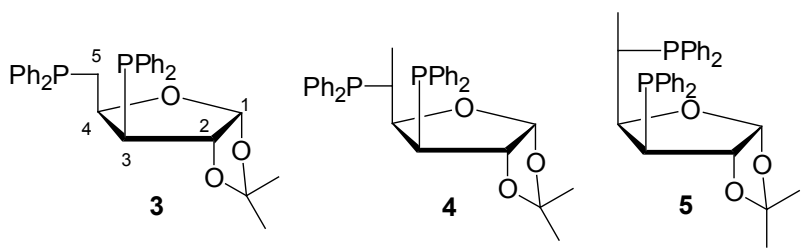

Scheme 2

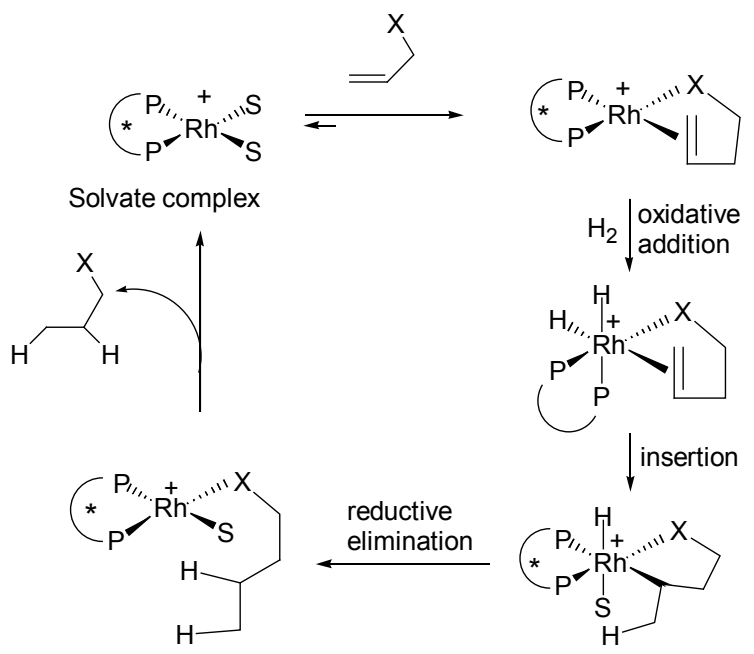

Scheme 3

环糊精是由多个葡萄糖组成的环状低聚物, 具有很 强的分子识别功能, 功能化环糊精金属复合物用于催化 不对称反应具有潜在的诱导手性的能力. Jia 等 ${ }^{[22]}$ 在 $\beta$ 环糊精结构中引入二苯基膦官能团制得了含膦环糊精 配体 6 (Scheme 4), 将配体 6 用于 Rh 催化的底物为 $\alpha$ 乙酰氨基肉桂酸(酯)、 $\alpha$-乙酰氨基丙烯酸(酯)及衣康酸 (酯)的不对称氢化反应, 实验结果发现底物为衣康酸时 反应所得产物具有很高的对映选择性, 但是其它底物反 应所得产物的对映选择性都较低. 由于配体 6 与金属复 合物在水中溶解性较差所以该反应只能在有机溶剂(如 甲醇、乙醇、二氯甲烷、四氢呋喃等)中进行, 实验结果 也发现不同的溶剂也对产物的对映选择性产生影响, 该 反应在甲醇中所得产物具有最高的对映选择性.

\section{2 亚膦酸酯糖基配体}

含二亚膦酸酯结构的糖基配体 7 (Scheme 5)也被用 于烯烃不对称氢化反应中 ${ }^{[23}$ 35], 如将配体 7 用于不饱和 氨基酸氢化反应时, 所得产物的 $e e$ 值最高可达 $99 \%$. 后 来进一步的研究发现配体 7 中磷原子相连的苯基上连有

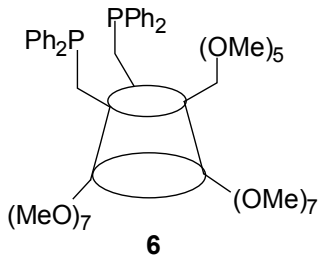

Scheme 4

给电子基团时所得产物具有较高的对映选择性，而连有 吸电子基团时所得产物的对映选择性都较差(如反应中 使用配体 7a, $7 \mathbf{b}\left(\mathrm{R}^{\prime}=\mathrm{Ph}\right)$ 所得产物的 $e e$ 较高, 而使用 7f $7 \mathbf{g}$ 所得产物的 $e e$ 值都较低), 并且该反应所得产物主要 为 $S$ 构型.

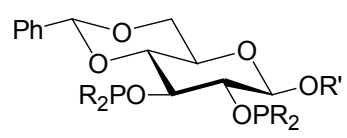

$$
7 \mathrm{R}^{\prime}=\mathrm{Me}, \mathrm{Ph}
$$
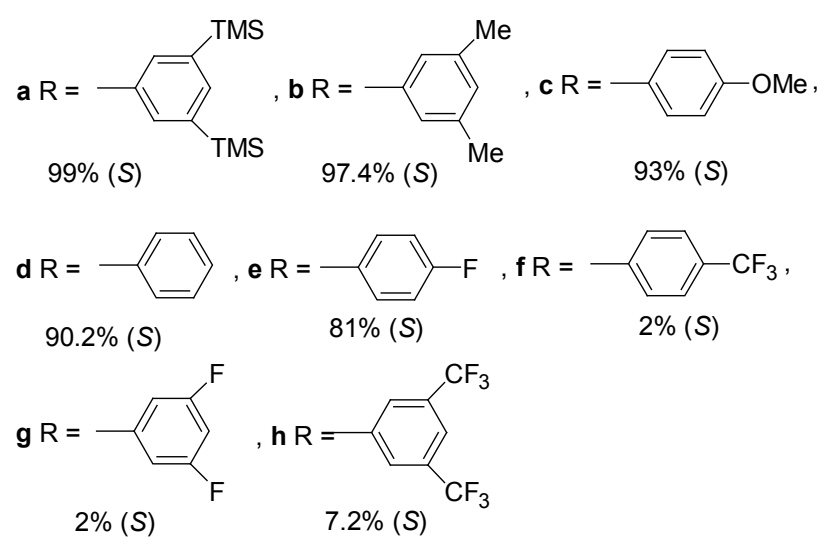

Scheme 5

RajanBabu 等 ${ }^{[32 ~ 34]}$ 合成了含二膦酸酯结构的配体 $\mathbf{8}$ (Scheme 6), 并将其用于不饱和氨基酸为底物的不对称 氢化反应中, 结果发现可以高对映选择性地得到 $R$ 构型 产物, 实验中考查了磷原子所连苯环结构对产物对映选 择性的影响: 苯环上连有给电子基团的配体(如 $\mathbf{8 a}, \mathbf{8 f}$ ) 用于上述反应时所得产物都具有很高的对映选择性，而 使用配体 $8 \mathbf{b}$ 时产物的对映选择性有所降低，但使用配 体 $8 \mathrm{c}, 8 \mathrm{~d}, 8 \mathrm{e}$ 时所得产物的对映选择性普遍很低, 这说 明对于该类反应而言糖基配体中磷原子相连的苯基连 有给电子基团有助于提高产物对映选择性.

Uemura 等 ${ }^{[36 ~ 38]}$ 设计合成了新型的含二亚膦酸酯 结构的糖基配体 9 10 (Scheme 7), 并将配体 9 10 用 于 $\mathrm{Rh}$ 催化的不饱和氨基酸的不对称氢化反应，结果发 现所得产物具有较高的对映选择性( ee 值最高可达 $84 \%$ ). 后来人们以 $D$-甘露醇为原料合成了配体 $\mathbf{1 1} \sim \mathbf{1 2}$ (Scheme 7)并将它们用于 $\mathrm{Rh}$ 催化的不对称氢化反应中, 实验结果发现配体 11 用在底物为不饱和氨基酸的反应 中所得产物 $e e$ 值最高可达 $96.7 \%$, 而配体 12 用在底物 


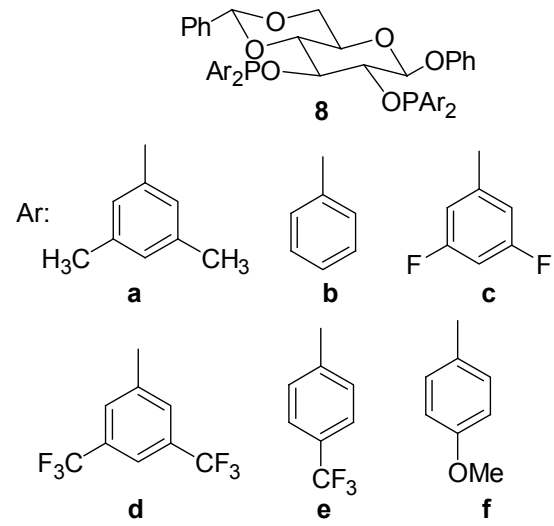

Scheme 6

为不饱和酮的反应中所得产物 $e e$ 值最高可达 $86 \% \%^{[39,40]}$. 最近一些新型含二亚膦酸酯呋喃糖基配体 $13 \sim 15$ (Scheme 7) 被设计合成出来并且被成功用于不对称氢化 反应中 ${ }^{[41,42]}$. 配体 $\mathbf{1 3} \sim \mathbf{1 4}$ 可由 $D$-木糖为原料合成, 将 它们用于底物为不饱和氨基酸的不对称氢化反应时可 高 $e e$ 值得到产物, 实验结果发现金属催化剂的种类以 及糖基中 C-3 的构型对产物对映选择性有很大影响, 如 实验中用 $\mathrm{Rh} /$ 配体 13 或者用 $\mathrm{Ir} /$ 配体 14 时产物的 $e e$ 值较 高, 但是用其它催化体系时产物 ee 值却很低. 配体 13 也被用于 Ir 催化的烯胺的不对称氢化反应中, 结果发现 产物的 $e e$ 值为中等 ${ }^{[43]}$. 配体 $\mathbf{1 5 a}$ 被成功用于 $\mathrm{Rh}$ 催化的 不饱和氨基酸的不对称氢化反应中(产物的 $e e$ 值最高可 达 93\%), 配体 15b 也被成功用于 Ir 催化的烯胺的不对 称氢化反应中(产物的 $e e$ 值最高可达 $70 \%)^{[22]}$.

\section{3 亚磷酸酯糖基配体}

Brunner ${ }^{[14]}$ 及 Selke 等 ${ }^{[44]}$ 最早报道了将含二亚磷酸 酯的糖基配体 16 17 (Scheme 8)用于不对称氢化反应, 但是他们的实验结果并不理想, 产物的对映选择性并不 高.

Reetz 等 ${ }^{[45]}$ 在研究含二亚磷酸酯糖基配体应用于不 对称氢化反应中取得了重要突破, 他们在 $D$-甘露醇结 构中引入不同结构的磷酸酯取代基合成了一系列糖基 配体 18 (Scheme 9)，这些配体被用于衣康酸衍生物等的 不对称氢化反应, 实验结果发现用配体 $18 \mathrm{e}$ 时反应产物 对映选择性最好，产物的 $e e$ 值可达到 $94.5 \%$.

最近，人们以 $D-(+)$ 木糖及 $D-(+)$ 葡萄糖为原料合 成了一系列的含二亚磷酸酯的呋喃型糖基配体 $19 \sim 24$ (Scheme 10), 并把这些配体用于 Rh 催化的不对称氢化 反应中(Eq. 1) ${ }^{[46 ~ 48]}$. 在以脱氢氨基酸为底物的反应中应 用这些配体后发现所得产物的 ee 值最高可大于 $99 \%$, 可见这类糖基配体具有很好的对映选择性; 随后人们又 研究了这些糖基配体中 $\mathrm{C}-3$ 及 $\mathrm{C}-5$ 的构型对产物 ee 值 的影响, 结果发现配体中糖基 C-3 构型对产物 $e e$ 值影响
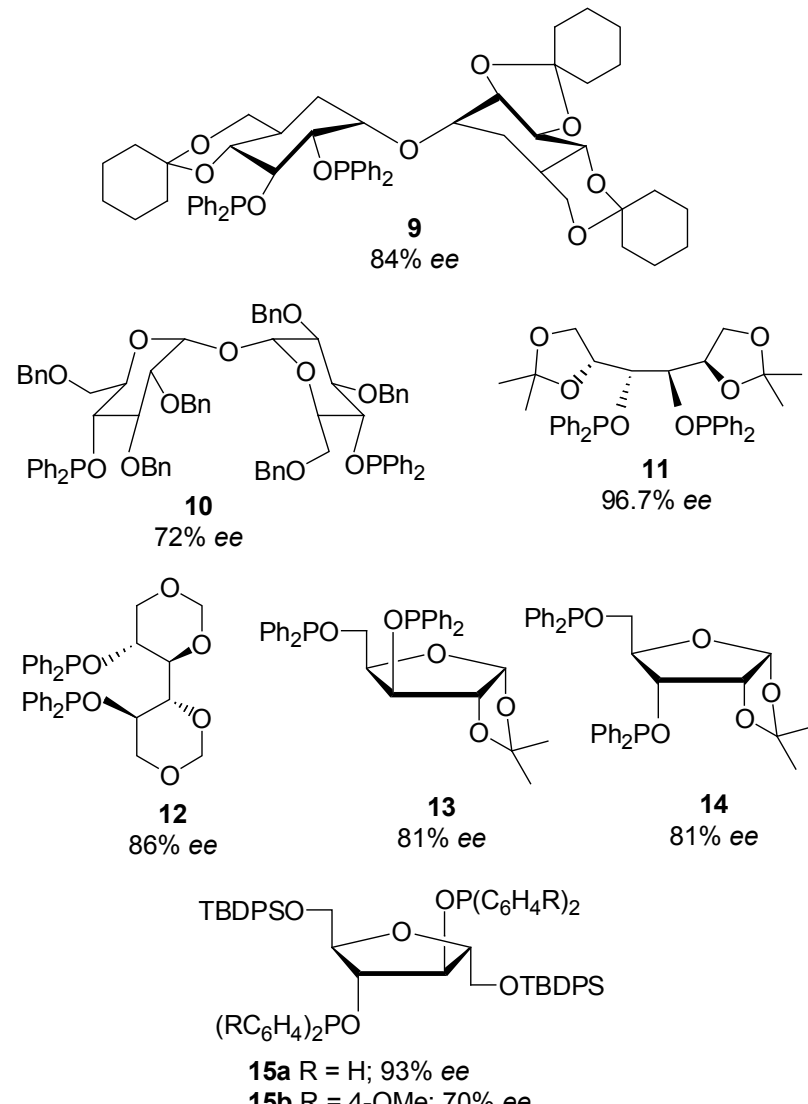

Scheme 7
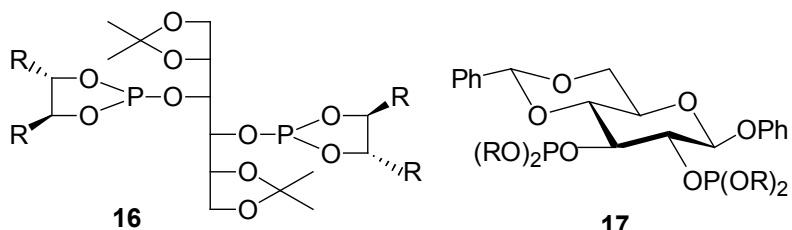

17

Scheme 8
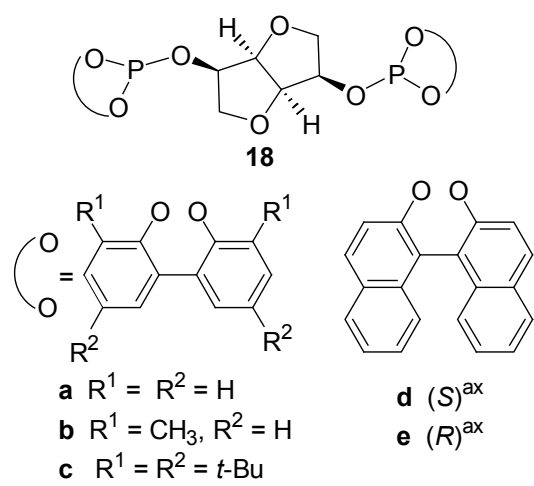

Scheme 9

很大而 C-5 构型对产物 $e e$ 值影响很小, 配体 21d 糖基中 C-3 及 C-5 均为 $R$ 构型在反应中表现出最好的对映选择 性, 在 Ir 催化的烯胺不对称氢化反应中使用配体 19 时 所得产物 $e e$ 值为中等. 

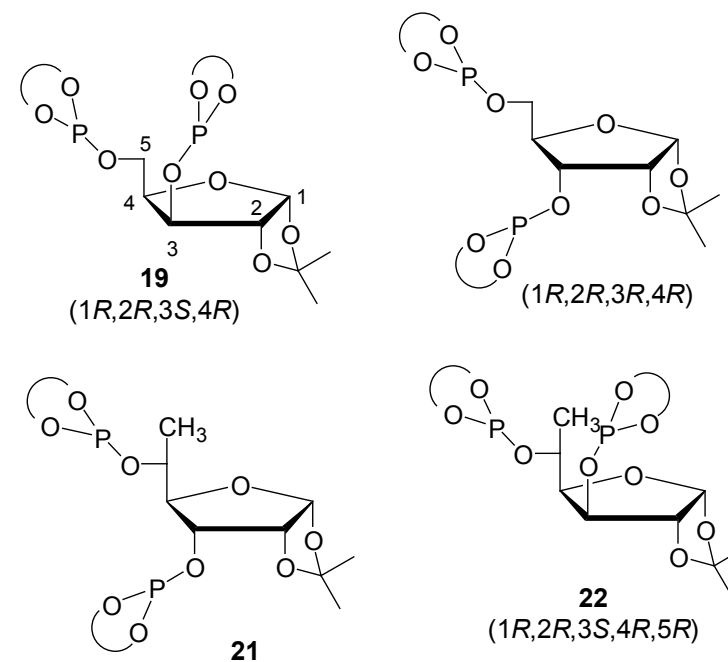

$(1 R, 2 R, 3 R, 4 R, 5 R)$

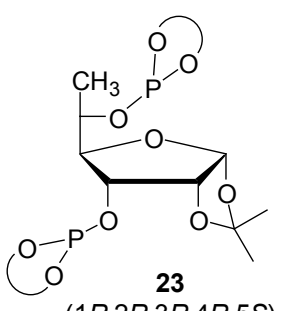

$(1 R, 2 R, 3 R, 4 R, 5 S)$

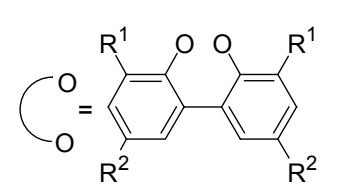

a $\mathrm{R}^{1}=\mathrm{R}^{2}=\mathrm{H}$

b $\mathrm{R}^{1}=\mathrm{R}^{2}=t-\mathrm{Bu}$

c $\mathrm{R}^{1}=t-\mathrm{Bu}, \mathrm{R}^{2}=\mathrm{OMe}$

d $\mathrm{R}^{1}=\mathrm{SiMe}_{3}, \mathrm{R}^{2}=\mathrm{H}$
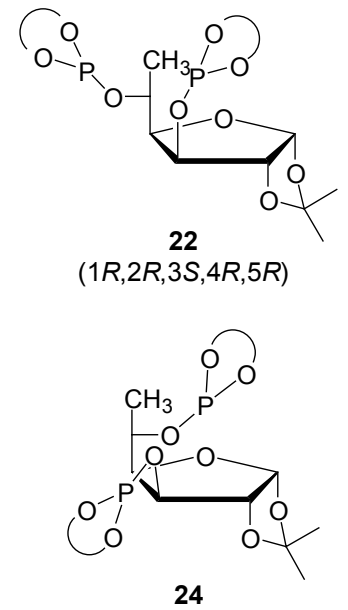

$(1 R, 2 R, 3 S, 4 R, 5 S)$

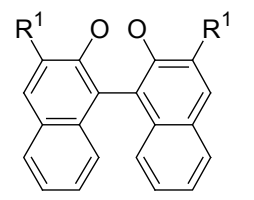

e $(S)^{a x} ; R^{1}=H$

f $(R)^{\text {ax. }} ; \mathrm{R}^{1}=\mathrm{H}$

g $(S)^{\mathrm{ax}} ; \mathrm{R}^{1}=\mathrm{SiMe}_{3}$

f $(R)^{\mathrm{ax}} ; \mathrm{R}^{1}=\mathrm{SiMe}_{3}$

\section{Scheme 10}

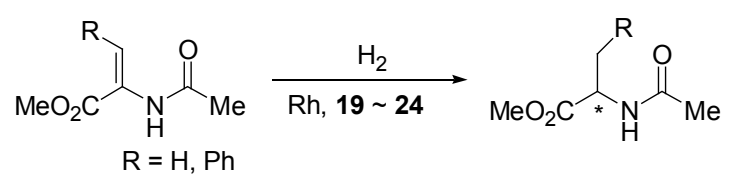

最近, Claver 等 ${ }^{[49]}$ 以 $D$-葡萄糖苷和 $D$-山梨醇为原料 合成了含二亚磷酸酯呋喃型糖基配体 $\mathbf{2 5} \sim 28$ (Scheme 11), 其结构与配体 15 类似. 这些配体被用于 $\mathrm{Rh}$ 催化的 一些丙烯酸盐衍生物为底物的不对称氢化反应中, 实验 结果发现这些配体的对映选择性比配体 15 低很多, 产 物的 $e e$ 值最高为 $57 \%$.

在过去的几十年中人们在不对称氢化反应中应用 的糖基配体研究最多的是二齿磷配体, 但是最近 Reetz 等 ${ }^{[50]}$ 将单齿磷配体成功地用于 $\mathrm{Rh}$ 催化的不对称氢化反 应中, 他们将二齿亚磷酸酯配体 18 进行改造得到单齿 亚磷酸酯配体 29 (Scheme 12), 实验结果发现后者应用
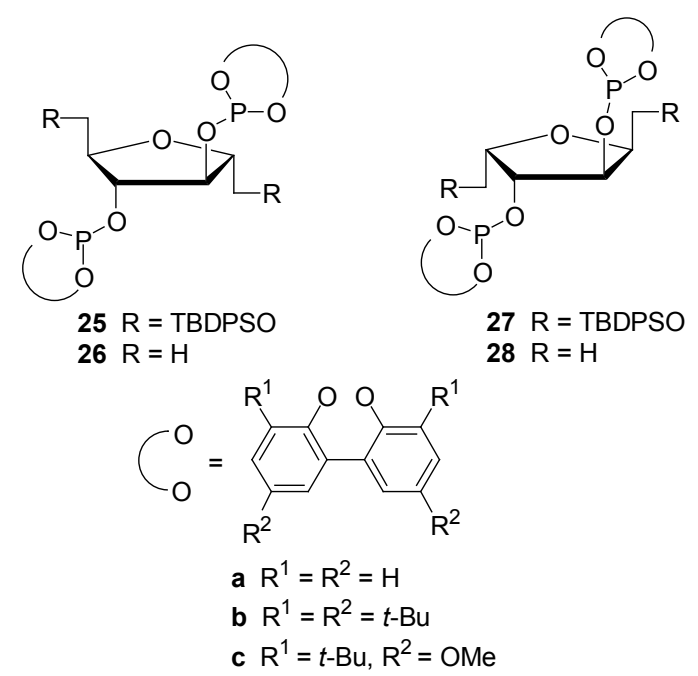

Scheme 11

于实验中的对映选择性与前者相当. 随后 Reetz 的研究 小组 ${ }^{[51]}$ 与 Chen 等 ${ }^{[52,53]}$ 分别对单齿亚磷酸酯配体做了深 入的研究，他们合成了一些有效的单齿亚磷酸酷配体并 将其用于 $\mathrm{Rh}$ 催化的不同底物的不对称氢化反应, 如 Reetz 等 ${ }^{[51]}$ 报道了将配体 30 34 (Scheme 13) 用于 $R h$ 催 化以不饱和羒酸酯为底物的不对称氢化反应(Eq. 2), 对 比不同配体后发现配体中二菜酚基团与糖基中手性碳 原子会协同影响产物的对映选择性，配体 30b 用于反应 所得产物对映选择性最好(产物 ee 值最高可达 94\%). Chen 等 ${ }^{[54,55]}$ 将配体 30 34 及配体 35 38 (Scheme 13) 用于 $\mathrm{Rh}$ 催化的以脱氢氨基酸(产物 $e e$ 值最高可达 98.4\%)以及以烯胺(产物 $e e$ 值最高可达 99.6\%)为底物的 不对称氢化反应. 实验结果表明糖基中 C-3 构型对产物 对映选择性有很大影响, 如配体 $\mathbf{3 1}$ 与 $\mathbf{3 6}$ (糖基中 C-3 为 $R$ 构型)比配体 $\mathbf{3 0}$ 与 $\mathbf{3 5}$ (糖基中 C-3 为 $S$ 构型)在实验中 表现出更高的对映选择性; 还表明配体中二萗酚基团与 糖基中手性碳原子会协同影响产物的对映选择性.
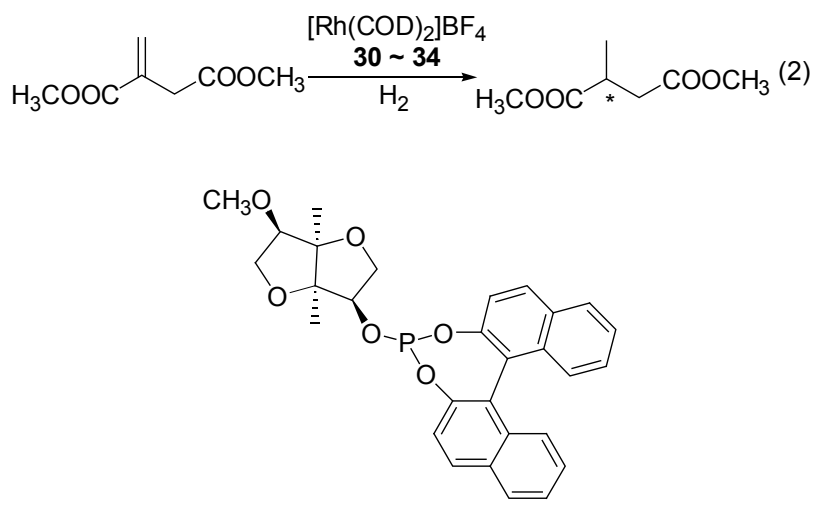

29a $(S)^{\text {ax }} 97.8 \%(S)$

29b $(R)^{\text {ax }} 95.2 \%(R)$

Scheme 12 


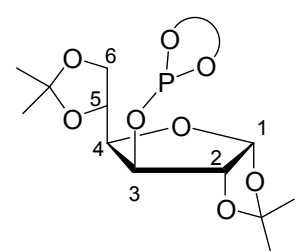

30

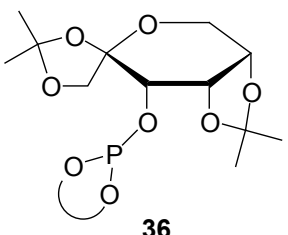

36

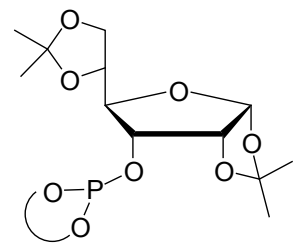

31<smiles>COC1COC(c2ccccc2)O[C@H]1OP1OCCO1</smiles>

37

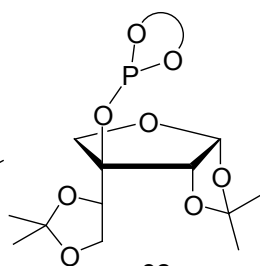

32

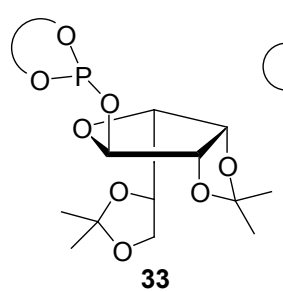

33

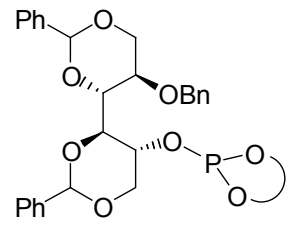

38

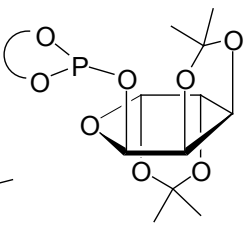

34

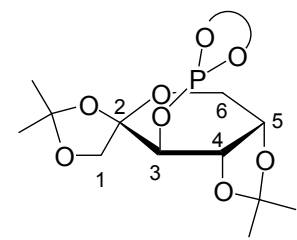

35

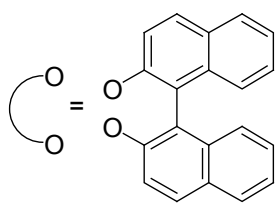

a $(S)^{a x}$

b $(R)^{a x}$

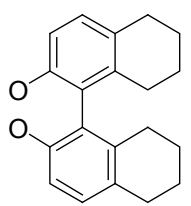

c $(R)^{\mathrm{ax}}$

Scheme 13

最近, Huang 等 ${ }^{[56]}$ 以 $D$-甘露醇为原料合成了手性离 子型单亚磷酸酯糖基配体 39a 39e 及双亚磷酸酯糖基 配体 40 (Scheme 14), 这些配体在空气中稳定, 在研究 溶剂性时发现它们仅溶于二氯甲烷、四氢呋喃及常见的 离子液体中; 这些配体被用于 $\mathrm{Rh}$ 催化的不同底物的不 对称氢化反应中(如以不同结构烯酰胺为底物时所得产 物的 $e e$ 值一般大于 $99 \%$; 以不饱和氨基酸酯为底物时 产物 $e e$ 值最高可达 99\%) (Eq. 3), 结果发现这些糖基配 体具有极高的对映选择性. 这些配体使用方便, 容易回 收, 在重复使用 10 次之后并未发现其催化活性有明显 的降低.

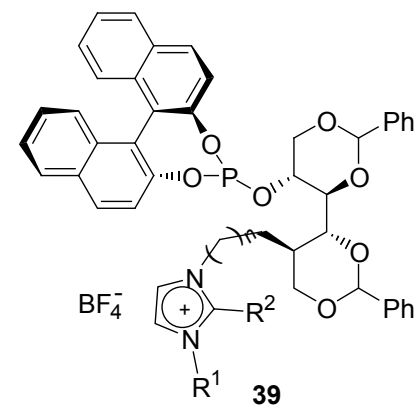

a $\mathrm{R}^{1}=\mathrm{Me} ; \mathrm{R}^{2}=\mathrm{H} ; n=4$ b $\mathrm{R}^{1}=\mathrm{Me} ; \mathrm{R}^{2}=\mathrm{Me} ; n=4$ $\mathrm{cR}^{1}=n-\mathrm{Bu} ; \mathrm{R}^{2}=\mathrm{H} ; n=4$ $\mathbf{d ~ R}^{1}=\mathrm{Me} ; \mathrm{R}^{2}=\mathrm{H} ; n=6$ e $\mathrm{R}^{1}=\mathrm{Me} ; \mathrm{R}^{2}=\mathrm{H} ; n=12$

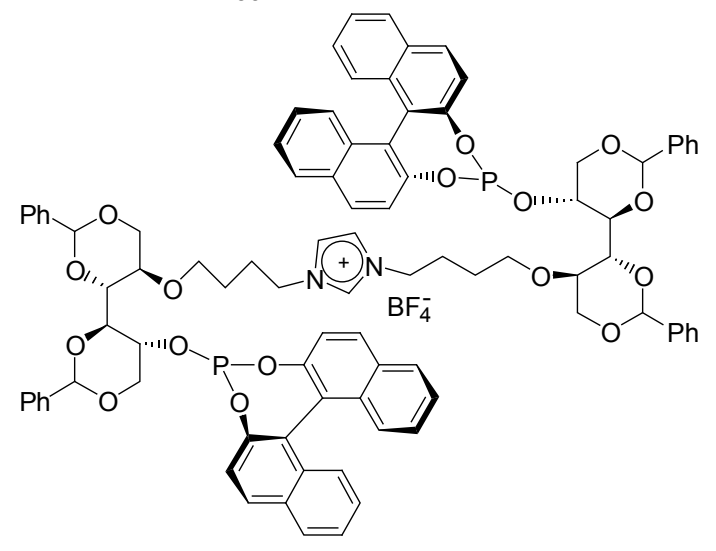

40

Scheme 14

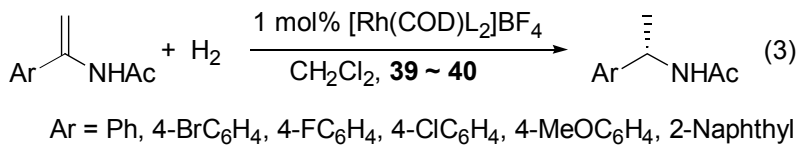

\section{4 多供体糖基配体}

在不对称氢化反应中应用的糖基配体除了含二膦、 二亚膦酸酯、二亚磷酸酯结构之外，人们还将其它不同 结构的给电子基团(如膦-亚磷酸酯、亚磷酸酯-亚磷酰 胺、亚磷酸酯-噁唑啉、膦酸-硫醚等)引入到糖基配体 中.

\subsection{P-P'配体}

最早报道的含膦-膦酸结构的糖基配体是以 $D$-木糖 苷为原料合成的配体 $\mathbf{4 1}{ }^{[57]}$ 以及以 $L-\alpha, \alpha$-海藻糖为原料 合成的配体 $\mathbf{4 2}^{[58]}$ (Scheme 15), 配体 $\mathbf{4 1}$ 被用于 $\mathrm{Rh}$ 催化 的以衣康酸为底物的反应中, 发现所得产物具有中等的 对映选择性, 实验中还将配体 41 与不含糖基但具有二 菜酚结构的配体活性进行了比较, 结果后者的对映选择 性好于前者; 配体 42 与铑构成阳离子配合物用于催化 水相中的脱氢氨基酸(酯)为底物的不对称氢化反应，底 物结构对产物对映选择性有很大影响(如底物 $\beta$-碳上连 有苯与䒬时所得产物 $e e$ 值普遍较高, 而 $\beta$-碳上连有氢 原子时所得产物 $e e$ 值普遍较低), 该催化剂在水相使用 时可以回收重复利用.

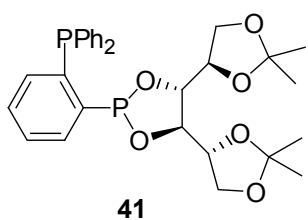<smiles>OC[C@H]1O[C@@H](O[C@H]2O[C@H](CO)[C@H](P)[C@@H](O)[C@H]2O)[C@H](O)[C@@H](O)[C@@H]1O</smiles>

Scheme 15

Claver 等 ${ }^{[59,60]}$ 将膦及亚磷酸同时引入糖基结构中得 
到了另一类含磷配体 43a 43e (Scheme 16), 这些配体 被用于 $\mathrm{Rh}$ 催化的以一些 $\alpha, \beta$-不饱和羒酸衍生物为底物 的不对称氢化反应, 在非常温和的条件下高对映选择性 地得到产物(产物 $e e$ 值最高可大于 $99 \%$ ). 这些配体中因 含有不同的亚磷酸基团而表现出不同的对映选择性, 实 验结果表明配体 $43 b$ 效果最好, 这可能与该配体二苯酚 基团中含有空间位阻很大的叔丁基有关, 表明配体中二 酚结构对产物的构型有很大影响, 如在反应中应用二荟 酚基团为 $R$ 构型的配体 43d 时所得产物以 $R$ 构型为主 (产物 $e e$ 值为 97.6\%); 而应用二萗酚基团为 $S$ 构型的配 体 43e 时所得产物以 $S$ 构型为主 (产物 $e e$ 值为 $98.3 \%$ ). 后 来他们在 $D$-木糖中引入了亚磷酸与亚磷酰胺基团得到 了配体 $\mathbf{4 4} \sim \mathbf{4 5}^{[61]}$ (Scheme 16), 他们在结构上与配体 19、20、43 比较相似, 由于在糖基 C-5 上引入了亚磷酰 胺基团配体 44 45 用于 $\mathrm{Rh}$ 催化的不对称氢化反应中表 现出了很高的对映选择性(反应中使用配体 44b 时产物 的 ee 值可达 99\%). 与配体 $\mathbf{2 5} \sim \mathbf{2 6}$ 相似的配体 $\mathbf{4 6}$ (Scheme 16) 被合成出来, 其中配体 $\mathbf{4 6 f}$ 被成功用于 Ir 催 化的以酮亚胺衍生物为底物的不对称氢化反应中, 所得 产物的 $e e$ 值达到 $73 \%{ }^{[62]}$.
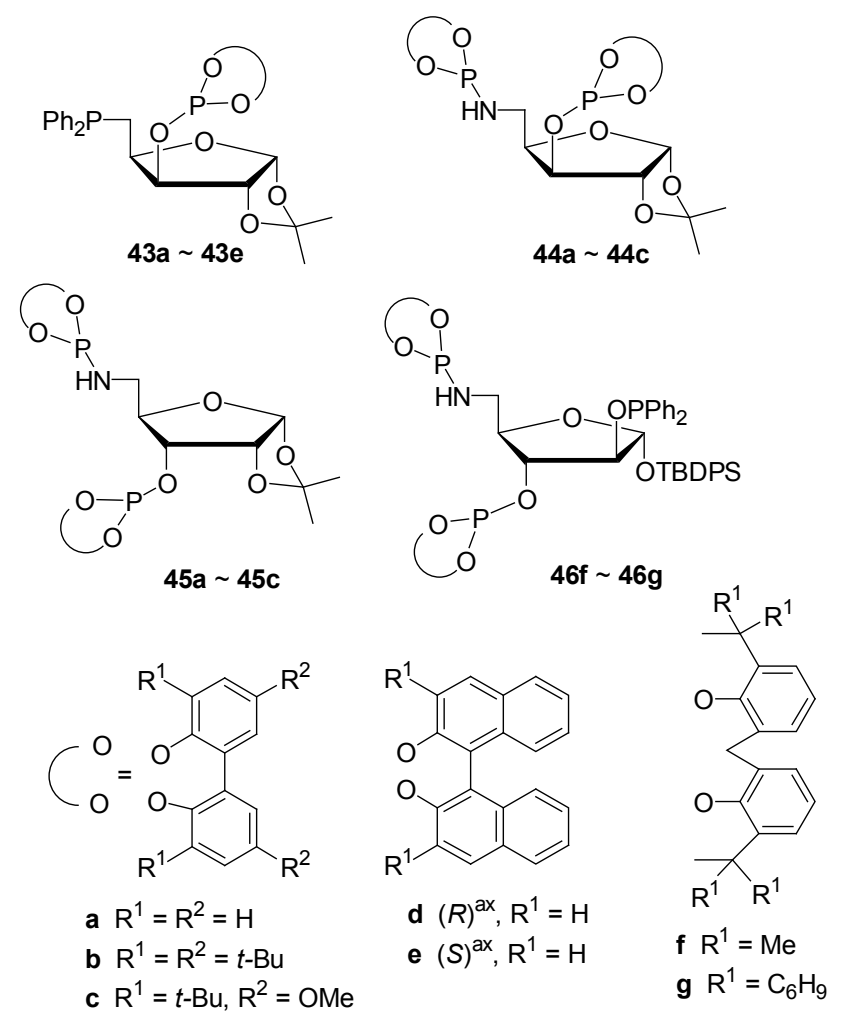

a $\mathrm{R}^{1}=\mathrm{R}^{2}=\mathrm{H}$

b $\mathrm{R}^{1}=\mathrm{R}^{2}=t-\mathrm{Bu}$

c $\mathrm{R}^{1}=t-\mathrm{Bu}, \mathrm{R}^{2}=\mathrm{OMe}$

Scheme 16

\subsection{P-N 配体}

寻找新型的更加有效的配体一直是不对称催化研 究的热点问题, 最近 Andersson 等 ${ }^{[63 \sim 64]}$ 合成了以 $\mathrm{P}, \mathrm{N}$ 原 子作为配位原子的新型配体 47 51 (Scheme 17), 这些
配体中含有磷酸与噁唑啉结构, 可以 $D$-氨基糖为原料 通过简单步骤合成, 并可以通过改变恶唑啉与芳基所连 基团进行修饰，它们被成功用于 Ir 催化的以一些苯乙烯 衍生物为底物的不对称氢化反应中(Eq. 4), 这些配体表 现出很高的活性与对映选择性, 但是反应底物中具有 1,2-二苯乙烯结构时比具有苯乙烯时所得产物普遍具有 更高的对映选择性, 实验中将含磷酸-噁唑啉糖基配体 与结构相似的含膦-噁唑啉糖基配体的活性进行了比较, 发现前者在实验中表现出了更高的对映选择性. 这类配 体的出现为设计新型的糖基配体提供了新的思路, Andersson 等 ${ }^{[64]}$ 对这类配体用于铱催化的不对称氢化反 应中的反应机理做了探讨(Scheme 18).
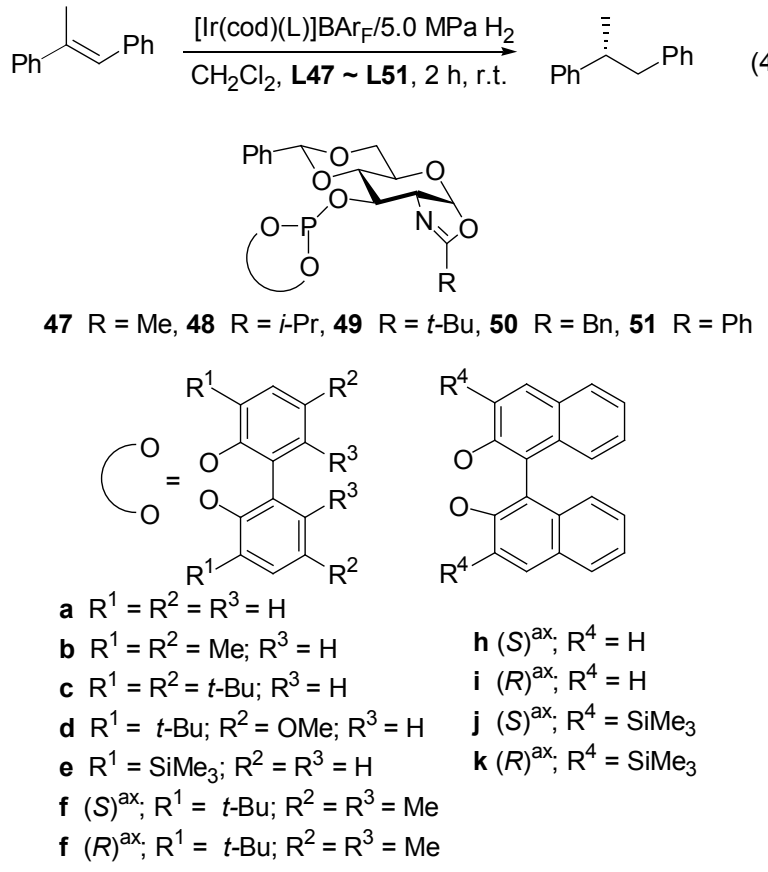

Scheme 17

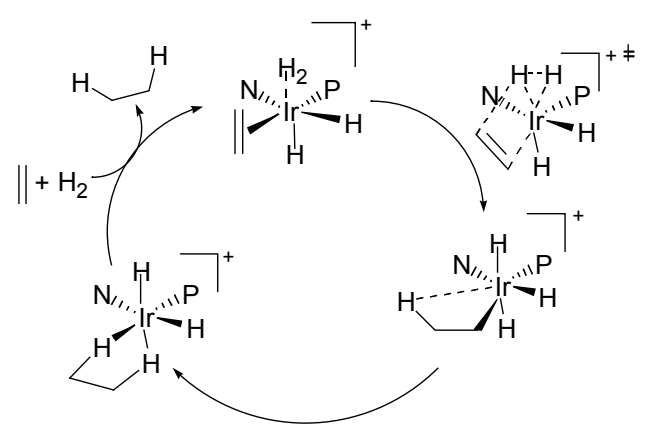

Scheme 18

最近, Diéguez 等 ${ }^{[65]}$ 将吡啶与亚磷酸酯共同引入糖 基结构之中得到了配体 52 53 (Scheme 19), 这些配体 被用于 Ir 催化的不对称氢化反应中(Eq. 5), 发现糖基中 C-2 或 C-3 所连的亚磷酸酯基的位置、结构以及它们的 构型都会对反应产物的对映选择性产生影响, 以 $E-2-$ 
(4-甲氧基苯基)-2-丁烯为底物的反应中应用配体 52a 和 52c 时所得产物的 $e e$ 值最高可达 $90 \%$, 这可能与配体中 糖基 C-2 上的亚磷酸酯基与具有较大空间位阻作用并且 为 $R$ 构型二苯基能产生有效的协同作用有关.

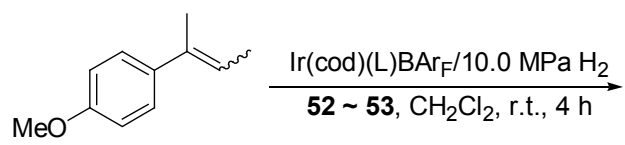<smiles>CCC(C)c1ccc(OC)cc1</smiles>
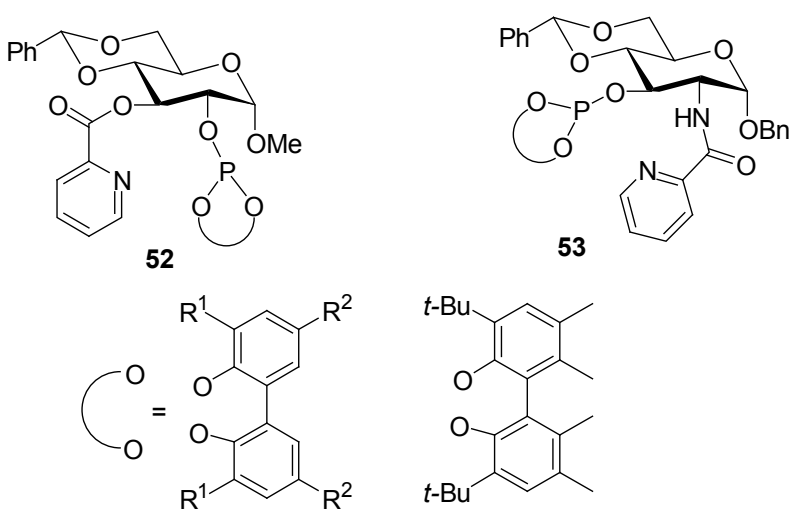

a $\mathrm{R}^{1}=\mathrm{R}^{2}=t-\mathrm{Bu}$

c $(R)^{\mathrm{ax}}$

b $\mathrm{R}^{1}=\mathrm{SiMe}_{3}, \mathrm{R}^{2}=\mathrm{H}$

d $(S)^{\mathrm{ax}}$

\section{Scheme 19}

\subsection{P-S 配体}

最近, $\mathrm{P}, \mathrm{S}$ 作为配位原子的糖基配体 $\mathbf{5 4} \sim \mathbf{6 0}$ (Scheme 20) 已被设计并合成出来, 这些配体与 $\mathrm{Rh}$ 构成 环状配合物(它们具有单一的构型, 其构型已通过核磁 共振方法加以确定)作为催化剂已经被成功用于催化不 对称氢化反应 ${ }^{[66 ~ 68]}$. 在以某些脱氢氨基酸酯为底物的 反应中应用这些配体时发现: 反应中使用配体 54 时并 未得到加氢产物; 使用配体 54 时即使在氢气压力很高 的条件下所得产物的对应选择性也很差; 使用配体 56 58 时, 可以高产率与高 $e e$ 值地得到加氢产物(其中 使用配体 56 效果最好, 所得产物产率为 $100 \%, e e$ 值为 94\%). 实验结果表明糖基中 C-1, C-2 的结构与 S 原子所 连基团对产物的对映选择性有着非常重要的影响(比如 在反应中配体 55 的对映选择性很差, 但是将其结构中 硫原子相连的芳基用空间位阻很大的叔丁基取代得到 的配体 56 对映选择性却很高), 在设计配体结构时应多 注意这方面问题.

\section{5 结束语}

不对称催化氢化反应是目前有机化学研究的一个
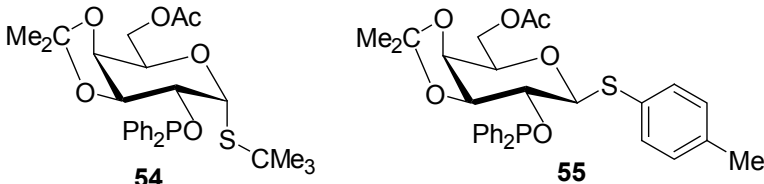

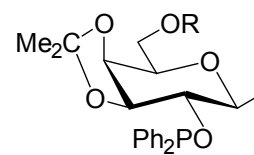

$56(\mathrm{R}=\mathrm{Ac})$

$57(\mathrm{R}=\mathrm{H})$

$58(\mathrm{R}=\mathrm{TBDMS})$

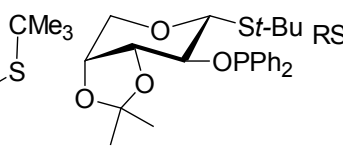

59

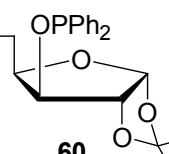
a $\mathrm{R}=\mathrm{Ph}$, b $\mathrm{R}=\mathrm{Me}$, c $\mathrm{R}=i-\mathrm{Pr}$

Scheme 20

热点课题，一些具体的反应在工业化生产中已有应用的 先例. 手性配体及手性催化剂一直是不对称催化研究的 重要课题，但是没有一种配体是通用的，因此发展新型 高效的手性配体仍然是今后研究的热点问题; 近年来手 性糖类化合物的合成与应用日益受到人们的关注，糖类 结构中含有多个手性中心，通过适当修饰已被广泛作为 不对称氢化反应的手性配体，并且取得了良好的效果， 可以预言的是对糖类化合物进行进一步修饰得到应用 于不对称氢化反应中的催化效率与选择性更高、适应范 围更广的新型的糖基配体仍是今后研究的一个热点课 题.

\section{References}

[1] Hollinsworth, R. I.; Wang, G. Chem. Rev. 2000, 100, 4267.

[2] Boysen, M. M. K. Chem. Eur. J. 2007, 13, 8648.

[3] Zhang, Z.-F.; Xie, F.; Yang, B.; Yu, H.; Zhang, W.-B. Chin. J. Org. Chem. 2011, 31, 429 (in Chinese). (张振锋, 谢芳, 杨波, 余焓, 张万斌, 有机化学, 2011, 31, 429.)

[4] Wang, Y.-D.; Wang, F.; Miao, Z.-W.; Chen, R.-Y. Prog. Chem. 2008, 20, 1923 (in Chinese).

(王亚丹, 王飞, 苗志伟, 陈茹玉, 化学进展, 2008, 20, 1923.)

[5] Nagel, U.; Kinzel, E.; Andrade, J.; Prescher, G. Chem. Ber. 1986, 119,3326

[6] Nagel, U.; Rieger, B. Organometallics 1989, 8, 1534.

[7] Lafont, D.; Sinou, D.; Descotes, G. J. Organomet. Chem. 1979, $169,87$.

[8] Bakos, J.; Heil, B.; Markó, L. J. Organomet. Chem. 1983, 253, 249.

[9] Johnson, T. H.; Rangarajan, G. J. Org. Chem. 1980, 45, 62.

[10] Li, C.; Bernet, B. B.; Vasella, A.; Broger, E. A.; Meili, A. Carbohydr. Res. 1991, 149.

[11] Choudary, B. M.; Sarma, M. R.; Prasad, A. D.; Narender, N. Indian J. Chem. B 1994, 33, 152.

[12] Gilbertson, S. R.; Chang, C. W. T. J. Org. Chem. 1995, 60,6226.

[13] Brunner, H.; Sievi, R. J. Organomet. Chem. 1987, 328, 71.

[14] Brunner, H.; Pieronczyk, W. J. Chem. Res., Synop. 1980, 76.

[15] Yamashita, M.; Hiramatsu, K.; Yamada, M.; Suzuki, N.; Inokawa, S. Bull. Chem. Soc. Jpn. 1982, 55, 2917.

[16] Saito, S.; Nakamura, Y.; Morita, Y. Chem. Pharm. Bull. 1985, 33, 5284.

[17] Iida, A.; Yamashita, M. Bull. Chem. Soc. Jpn. 1988, 61, 2356.

[18] Lemaux, P.; Massonneau, V.; Simonneaux, G. J. Organomet. 
Chem. 1985, 284, 101.

[19] Pàmies, O.; Net, G.; Ruiz, A.; Claver, C. Eur. J. Inorg. Chem. 2000, 2011.

[20] Diéguez, M.; Pamies, O.; Ruiz, A.; Castillon, S.; Claver, C. Tetrahedron: Asymmetry 2000, 11, 4701.

[21] Gridenev, I. D.; Higashi, N.; Asakura, K.; Imamoto, T. J. Am. Chem. Soc. 2000, 122, 7183.

[22] Wong, Y. T.; Yang, C.; Ying, K. C.; Jia, G. Organometallics 2002, 21,1782 .

[23] Cullen, W. R.; Sugi, Y. Tetrahedron Lett. 1978, 19, 1635.

[24] Jackson, R.; Thompson, D. J. J. Organomet. Chem. 1978, 159, C29.

[25] Selke, R. React. Kinet. Catal. Lett. 1979, 10, 135.

[26] Sinou, D.; Descotes, G. React. Kinet. Catal. Lett. 1980, 14, 463.

[27] Selke, R.; Pracejus, H. J. Mol. Catal. 1986, 37, 213.

[28] Selke, R. J. Prakt. Chem. 1987, 4, 717.

[29] Selke, R. J. Organomet. Chem. 1989, 370, 249.

[30] Selke, R.; Scwarze, M.; Baudishi, H.; Grassert, I.; Michalik, M.; Oehme, G.; Costisella, B. J. Mol. Catal. 1993, 84, 223.

[31] Selke, R.; Ohff, M.; Riepe, A. Tetrahedron 1996, 52, 15079.

[32] RajanBabu, T. V.; Ayers, T. A.; Casalnuovo, A. L. J. Am. Chem. Soc. 1994, 116, 4101.

[33] RajanBabu, T. V.; Ayers, T. A.; Halliday, G. A.; You, K. K.; Calabrese, J. C. J. Org. Chem. 1992, 62, 6012.

[34] Ayers, T. A.; RajanBabu, T. V. US 5510507, 1996 [Chem. Abstr. 1995, 124, 9449].

[35] Yan, Y. Y.; RajaBabu, T. V. J. Org. Chem. 2001, 66, 3277.

[36] Yonehara, K.; Hashizume, T.; Ohe, K.; Uemura, S. Bull. Chem. Soc. Jpn. 1998, 71, 1967.

[37] Yonehara, K.; Ohe, K.; Uemura. S. J. Org. Chem. 1999, 64, 9381.

[38] Yonehara, K.; Hashizume, T.; Mori, K.; Ohe, K.; Uemura, S. J. Org. Chem. 1999, 64, 5593.

[39] Chen, Y.; Li, X.; Tong, S.; Choi, M. C. K.; Chan, A. S. C. Tetrahedron Lett. 1999, 40, 957.

[40] Naili, S.; Suisse, I.; Mortreux, A.; Agbossou, F.; Ali, M. A.; Karim, A. Tetrahedron Lett. 2000, 41, 2867.

[41] Guimet, E.; Diéguez, M.; Ruiz, A.; Claver, C. Tetrahedron: Asymmetry 2004, 15, 2247.

[42] Aghmiz, M.; Aghmiz, A.; Diaz, Y.; Masdeu-Bulto, A. M.; Claver, C.; Castillon, S. J. Org. Chem. 2004, 69, 7502.

[43] Guiu, E.; Munoz, B.; Castillon, S.; Claver, C. Adv. Synth. Catal. 2003, 345, 169.

[44] Kadyrov, R.; Heller, D.; Selke, R. Tetrahedron: Asymmetry 1999, 9, 329.
[45] Reetz, M. T.; Neugebauer, T. Angew. Chem. Int. Ed. 1999, 38, 179.

[46] Pàmies, O.; Net, G.; Ruiz, A.; Claver, C. Eur. J. Inorg. Chem. 2000, 1287.

[47] Pàmies, O.; Net, G.; Ruiz, A.; Claver, C. Tetrahedron: Asymmetry 2000, 11, 1097.

[48] Diéguez, M.; Ruiz, A.; Claver, C. J. Org. Chem. 2002, 67, 3796.

[49] Axet, M. R.; Benet-Buchholz, J.; Claver, C.; Castillon, S. Adv. Synth. Catal. 2007, 349, 1983.

[50] Reetz, M. T.; Mehler, G. Angew. Chem., Int. Ed. 2000, 39, 3889.

[51] Reetz, M. T.; Goosen, L. J.; Meiswinkel, A.; Paetzol, J.; Jensen, J. F. Org. Lett. 2003, 5, 3099.

[52] Huang, H.; Zheng, Z.; Luo, H.; Bai, C.; Hu, X.; Chen, H. Org. Lett. 2003, 5, 4137.

[53] Huang, H.; Liu, X.; Chen, S.; Chen, H.; Zheng, Z. Tetrahedron: Asymmetry 2004, 15, 2011.

[54] Huang, H.; Zheng, Z.; Luo, H.; Bai, C.; Hu, X.; Chen, H. J. Org. Chem. 2004, 69, 2335.

[55] Huang, H.; Liu, X.; Chen, H.; Zheng, Z. Tetrahedron: Asymmetry 2005, 16, 693.

[56] Zhao, Y.; Huang, H.; Shao, J.; Xia, C. Tetrahedron: Asymmetry 2011, 22, 769 .

[57] Reetz, M. T.; Gosberg, A. Tetrahedron: Asymmetry 1999, 10, 2129.

[58] Ohe, K.; Morioka, K.; Yonehara, K.; Uemura, S. Tetrahedron: Asymmetry 2002, 13, 2155.

[59] Pàmies, O.; Diéguez, M.; Net, G.; Ruiz, A.; Claver, C. Chem. Commun. 2000, 2383.

[60] Pàmies, O.; Dieguez, M.; Net, G.; Ruiz, A.; Claver, C. J. Org. Chem. 2001, 66, 8364.

[61] Diéguez, M.; Ruiz, A.; Claver, C. Chem. Commun. 2001, 2072.

[62] Guiu, E.; Aghmiz, M.; Diaz, Y.; Claver, C. Meseguer, B.; Militzer, C.; Castillon, S. Eur. J. Org. Chem. 2006, 627.

[63] Diéguez, M.; Mazuela, J.; Pàmies, O.; Verendel, J. J.; Andersson, P. G. J. Am. Chem. Soc. 2008, 130, 7208.

[64] Mazuela, J.; Norrby, P. O.; Andersson, P. G.; Pàmies, O.; Diéguez, M. J. Am. Chem. Soc. 2011, 133, 13634.

[65] Margalef, J.; Lega, M.; Ruffo, F.; Pàmies, O.; Diéguez, M. Tetrahedron: Asymmetry 2012, 23, 945.

[66] Khiar, N.; Suarez, B.; Stiller, M.; Valdivia, V.; Fernandez, I. Phosphorus, Sulfur Silicon Relat. Elem. 2005, 180, 1253.

[67] Khiar, N.; Navas, R.; Suarez, B.; Alvarez, E.; Fernandez, I. Org. Lett. 2008, 10, 3697.

[68] Guimet, E.; Dieguez, M.; Ruiz, A.; Claver, C. Dalton Trans. 2005, 2557.

(Li, L.) 